\title{
1 Improved modeling of sediment oxygen kinetics and fluxes in lakes and
}

\section{2 reservoirs}

4. Xiamei Man ${ }^{\dagger}$, Kevin A. Bierlein ${ }^{\ddagger}$, Chengwang Lei ${ }^{\dagger}$, Lee D. Bryant ${ }^{\S}$, Alfred Wüest ${ }^{\| \prime, \#}$, and John 5 C. Little

6. ${ }^{\dagger}$ Centre for Wind, Waves and Water, School of Civil Engineering, The University of Sydney,

7 Sydney, NSW 2006, Australia

8• $\quad$ Department of Civil and Environmental Engineering, Virginia Tech, 401 Durham Hall,

9 Blacksburg, Virginia, USA

$10 \S$ Department of Architecture and Civil Engineering, University of Bath, Bath, United Kingdom

11 "Eawag, Swiss Federal Institute of Aquatic Science and Technology, Surface Waters - Research

12 and Management, Kastanienbaum, Switzerland

13 \# Physics of Aquatic Systems Laboratory, Margaretha Kamprad Chair, ENAC-IEE-APHYS, Swiss

14 Federal Institute of Technology (EPFL), Lausanne, Switzerland

$15 *$ Corresponding email: jcl@vt.edu

\section{ABSTRACT}

18 To understand water quality degradation during hypoxia, we need to understand sediment oxygen

19 fluxes, the main oxygen sink in shallow hypolimnia. Kinetic models which integrate diffusion and 20 consumption of dissolved oxygen (DO) in sediments usually assume a downward flux of DO from

21 the sediment-water interface (SWI) with a zero-flux condition at the lower boundary of the oxic

22 sediment layer. In this paper, we separately account for the oxidation of an upward flux of reduced

23 compounds by introducing a negative flux of DO as a lower boundary condition. Using in situ

24 measurements in two lakes, kinetic models were fit to DO microprofiles using zero-order and first-

25 order kinetics with both zero and non-zero lower boundary conditions. Based on visual inspection

26 and goodness-of-fit criteria, the negative-flux lower boundary condition, $-0.25 \mathrm{~g} \mathrm{O}_{2} \mathrm{~m}^{-2} \mathrm{~d}^{-1}$, was

27 found to more accurately describe DO consumption kinetics. Fitted zero-order rate constants 28 ranged from $50-510 \mathrm{mg} \mathrm{L}^{-1} \mathrm{~d}^{-1}$ and first-order rate constants ranged from $60-400 \mathrm{~d}^{-1}$, which

29 agree well with prior laboratory studies. DO fluxes at the SWI calculated from the simulated This document is the accepted manuscript version of the following article: Man, X., Bierlein, K. A., Lei, C., Bryant, L., Wueest, A., \& Little, J. C. (2020). Improved modeling of sediment oxygen kinetics and fluxes in lakes and reservoirs. Environmental Science and Technology. https://doi.org/10.1021/acs.est.9b04831 
30 profiles with the negative-flux lower boundary condition also showed better agreement with the 31 observed DO fluxes than the simulated profiles with the zero-flux lower boundary condition. 


\section{TOC ART}

34

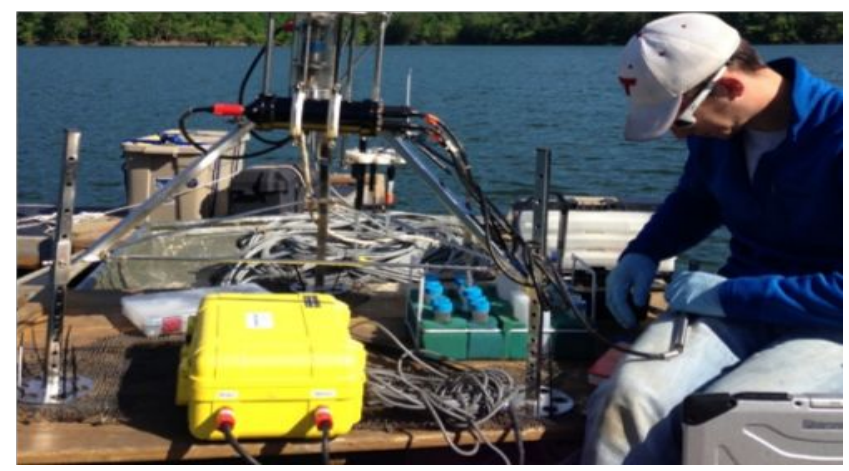

35 


\subsection{INTRODUCTION}

37 Hypoxia, often defined as dissolved oxygen (DO) $<2 \mathrm{mg} \mathrm{L}^{-1}$, commonly occurs in the deep waters of marine and freshwater systems during seasonal stratification ${ }^{1}$. In lakes and reservoirs, hypoxia usually leads to a lower redox potential in the sediments ${ }^{2}$, which can result in the reduction and dissolution of ferric and manganese oxides, decreasing their capacity to adsorb and retain nutrients. As a result, the concentration of nutrients (e.g., phosphorus and nitrogen) in the overlying water column increases ${ }^{3-5}$ which may exacerbate blooms of nuisance algae and cyanobacteria ${ }^{6-8}$. Algal and cyanobacterial blooms are significant concerns for lake and reservoir management because they stimulate eutrophication ${ }^{9}$, complicate water treatment processes and increase water treatment $\operatorname{costs}^{10}$. To make matters worse, some algae and cyanobacteria can be toxic ${ }^{11}$ (e.g., Karenia brevis), causing fish mortalities ${ }^{9}$ and restricting tourism in coastal areas ${ }^{12}$.

In the 1960s and 1970s, eutrophication stimulated by biologically available phosphorus was recognized as the primary cause of hypolimnetic hypoxia ${ }^{13}$ in lakes and reservoirs. However, it was later discovered that simply preventing the release of phosphorous from the sediment by artificial aeration or oxygenation ${ }^{10,14}$ did not eliminate hypolimnetic hypoxia as expected ${ }^{15,16}$. In

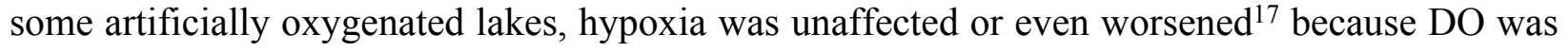
added to the water column but did not penetrate into the sediment, where reduced substances ( e.g., methane and ammonium) impacting hypolimnetic DO consumption are released ${ }^{18}$. Hypolimnetic DO demand and corresponding hypoxia are also influenced by DO consumption in the water column resulting from various chemical reactions and biological processes ${ }^{19}$, such as settling of organic matter ${ }^{20}$. To better understand the processes governing hypolimnetic DO consumption,

Sediment oxygen flux $\left(J_{O 2}\right)$ is usually the dominant sink for DO in the hypolimnion and usually comprises a majority of the DO demand in shallow water bodies. Processes on both sides of the sediment-water interface (SWI) influence $J_{O_{2}}{ }^{21}$. On the water-side, $J_{O 2}$ is controlled by the presence of a diffusive boundary layer (DBL) immediately above the SWI. Molecular diffusion

64 is the primary transport mechanism for DO through this thin, millimeter-scale layer, limiting the 65 rate of DO transport to the SWI. Turbulent flow in the bottom boundary layer generally controls the thickness of the DBL $\left(\delta_{D B L}\right)$, and is therefore an important factor controlling $J_{O 2}$ from the water- 
67 side of the $\mathrm{SWI}^{22}$. The concentration gradient of DO across the DBL also plays an important role, 68 as it provides the driving force for diffusive transport. On the sediment-side of the SWI, DO may 69 be transported deeper into the sediment porewater via diffusion or interstitial flow and is consumed 70 within the sediments by aerobic microbial respiration and chemical oxidation of reduced species 71 (i.e., manganese, iron, ammonium and methane). Müller et al. ${ }^{23}$ studied areal hypolimnetic 72 mineralization (AHM) rates in 21 lakes in Switzerland and France and found that rates varied 73 based on both sediment composition, depth of the hypolimnion and artificial oxygenation 74 conditions. Under quiescent near-sediment flows, water-side processes typically limit $J_{O 2}{ }^{24}$. 75 Alternatively, sediment-side chemical and biological processes requiring DO can limit $J_{O 2}$ under 76 turbulent conditions, when the supply of DO from the water column to the SWI exceeds the rate 77 at which it can be consumed or transported deeper into the sediment ${ }^{24}$. In the hypolimnion of most

78 lakes, turbulence near the SWI is low, meaning $J_{O 2}$ is primarily controlled on the water-side and 79 DO is readily consumed within the upper few millimeters of sediment. In artificially oxygenated 80 lakes and reservoirs, the oxygenation systems can enhance $J_{O_{2}}$ by increasing both turbulent mixing 81 and DO concentrations near the $\mathrm{SWI}^{25,26}$.

83 DO is consumed within the sediment by a broad range of processes driven by chemical and 84 biological reactions. When simulating DO consumption within the sediment, these processes are 85 usually lumped together into an apparent overall reaction rate, following zero-order, first-order, or 86 Monod kinetics ${ }^{19,22}$. Prior studies have investigated the transport of DO in the sediment by fitting 87 these kinetic models to DO microprofiles, or by measuring DO depletion rates in the water column above the sediment in different types of aquatic systems to calculate $J_{O 2}$ and estimate a rate constant. Brewer et al. ${ }^{27}$ tracked DO concentrations in the water above a dredged sediment sample

90 in laboratory incubations, assuming that DO consumption in the sediment followed first-order 91 kinetics. Likewise, Beutel et al. ${ }^{28}$ monitored DO depletion in sediment core incubations to 92 calculate $J_{O 2}$, also assuming first-order kinetics. Hall et al. ${ }^{29}$ measured DO depletion rates using 93 in situ flux chambers deployed in a Swedish fjord. They assumed zero-order kinetics, reporting a 94 rate constant of $1750 \mathrm{mg} \mathrm{L}^{-1} \mathrm{~d}^{-1}$ ( $\mathrm{L}$ refers to one litre of porewater in the sediment) at an in situ 95 temperature of $10^{\circ} \mathrm{C}$. Rasmussen and Jørgensen ${ }^{21}$ incubated sediment cores collected from Aarhus 96 Bay, Denmark, and measured DO microprofiles across the SWI using microsensors. They 97 considered zero-order and first-order kinetic models, finding zero-order kinetics to fit the 
98 microprofiles better than first-order. At an incubated temperature of $4^{\circ} \mathrm{C}$, they reported a fitted 99 zero-order rate constant of $83 \mathrm{mg} \mathrm{L}^{-1} \mathrm{~d}^{-1}$. House ${ }^{30}$ used dredged riverine sediments in a laboratory

100 flume to measure factors affecting DO penetration into the sediment, including flow velocity above 101 the sediment, sediment grain size, and organic matter content. DO was measured in the water and 102 sediment using microsensors, similar to the Rasmussen and Jørgensen ${ }^{22}$ study. House ${ }^{30}$ considered $^{-}$ 103 several kinetic models, including zero-order, first-order, and Monod kinetics. The study showed 104 that the simple zero-order model fit the data equally well or better than the more complex models. 105 Depending on the flow conditions above the SWI and the characteristics of the sediment, House 106 reported fitted zero-order rate constants ranging from $9.7-44 \mathrm{mg} \mathrm{L}^{-1} \mathrm{~d}^{-1}$ at an average temperature 107 of $18.4^{\circ} \mathrm{C}$.

These studies, while informative and worthwhile, are all somewhat limited by their methods or assumptions. A common approach is to only monitor DO in the water above the $\mathrm{SWI}^{19,27,28}$.

111 However, this does not capture the behavior of DO within the sediment and does not capture 112 processes driving the kinetics and actual DO consumption within the sediment. Studies performed 113 on sediment in laboratory incubations ${ }^{22,27,28,30}$ may disturb the sediment and SWI during the 114 dredging or coring process. Furthermore, laboratory conditions may not always be representative 115 of in situ conditions, particularly with respect to the critical control of turbulent flow in the water 116 overlying the sediment. The studies using microsensor measurements ${ }^{22,30}$ assumed that DO 117 profiles were at steady state when fitting kinetic models to the data, which is appropriate for a 118 laboratory study under controlled conditions that allow the incubated sediment to reach steady 119 state. However, Bryant et al. ${ }^{31}$ measured in situ DO microprofiles in a seiching lake and observed 120 large changes in the vertical DO distribution above the SWI and within the sediment on an hourly 121 timescale, highlighting the fact that ambient conditions in the field are typically quite variable and 122 that DO profiles in the sediment may never actually reach steady state.

124 Another key point is that almost all previous studies assumed zero-flux lower boundary conditions, 125 where the lower boundary was assumed to exist at the location where measurement terminated. 126 Interpretation of measured DO concentration profiles by Berg et al. ${ }^{32}$ addresses this by accounting 127 for increased DO consumption in the upper sediment zone due to bioturbation; however, the 128 specific contribution of the reduced species flux was not considered. 
130 A recent study that provides an innovative approach for upper sediment research is Müller et al. ${ }^{23}$.

131 Combining water-column, sediment, and porewater chemistry analyses and sediment-to-water flux

132 estimates in 11 eutrophic lakes, their study suggested a new method of describing sediment DO

133 consumption close to the lower boundary. One main outcome of their research is to demonstrate

134 that the estimated AHM rate $\left(0.25-0.49 \mathrm{~g} \mathrm{O}_{2} \mathrm{~m}^{-2} \mathrm{~d}^{-1}\right)$ in eutrophic lakes is induced by both

135 mineralization of organic material and a flux of reduced species propagating from the sediment

136 towards the SWI including methane, ammonium, nitrate $\left(\mathrm{NO}_{2}^{-}\right)$, manganese $(\mathrm{Mn}(\mathrm{II}))$ and iron (Fe

137 (II)). Their study is simplified compared to existing and more comprehensive models for sediment

138 diagenesis ${ }^{33}$, where the sediment is divided into an upper aerobic layer and a lower anaerobic layer.

139 More comprehensive models simulate organic matter mineralisation, nitrification, denitrification,

140 partitioning of hydrogen sulphide $\left(\mathrm{H}_{2} \mathrm{~S}\right)$ and oxidation, while the estimated AHM rate only

141 considers the two most essential hypolimnetic DO depletion processes. Despite the simplification,

142 the agreement between estimations and measurements of the AHM rates is striking. Although

143 their study did not directly focus on sediment DO microprofiles, this has significant implications

144 for the validity of the traditional sediment profile interpretation with the zero-flux boundary

145 condition and also shows the potential to simplify the modeling of sediment DO kinetics by

146 considering only the most essential biogeochemical processes impacting DO concentrations in the

147 upper sediment.

149 This paper focuses on how to better describe DO consumption close to the lower boundary of the oxic freshwater sediments. Transient DO profiles are modeled under two different lower boundary

151 conditions, but using the same governing equations. Simulated profiles with two lower boundary

152 conditions are then compared to determine which lower boundary condition is more appropriate.

153 Simulated sediment DO fluxes are also compared as they are critical parameters needed for

154 defining water quality and ecosystem health for lake and reservoir management. To our

155 knowledge, the current study is the first to fit kinetic models to obtain rate constants using DO

156 microprofiles measured in situ, rather than using laboratory incubations. It is also the first kinetic

157 study to consider the reaction of DO at the lower boundary with reduced species propagating 158 upward from deeper within the sediments, inspired by Müller et al. ${ }^{23}$. In this study, the oxidation 159 of the reduced species is assumed to be instantaneous at the bottom of the oxic sediment layer. 
160 Although DO consumption within the oxic sediment layer is partially related to reduced species

161 penetrating upward, there is evidence that the gradient of reduced species close to the bottom of 162 the oxic layer is higher than in other regions of the upper sediment ${ }^{34,35}$.

\subsection{METHODS}

\section{$165 \quad 2.1 \quad$ Study Sites}
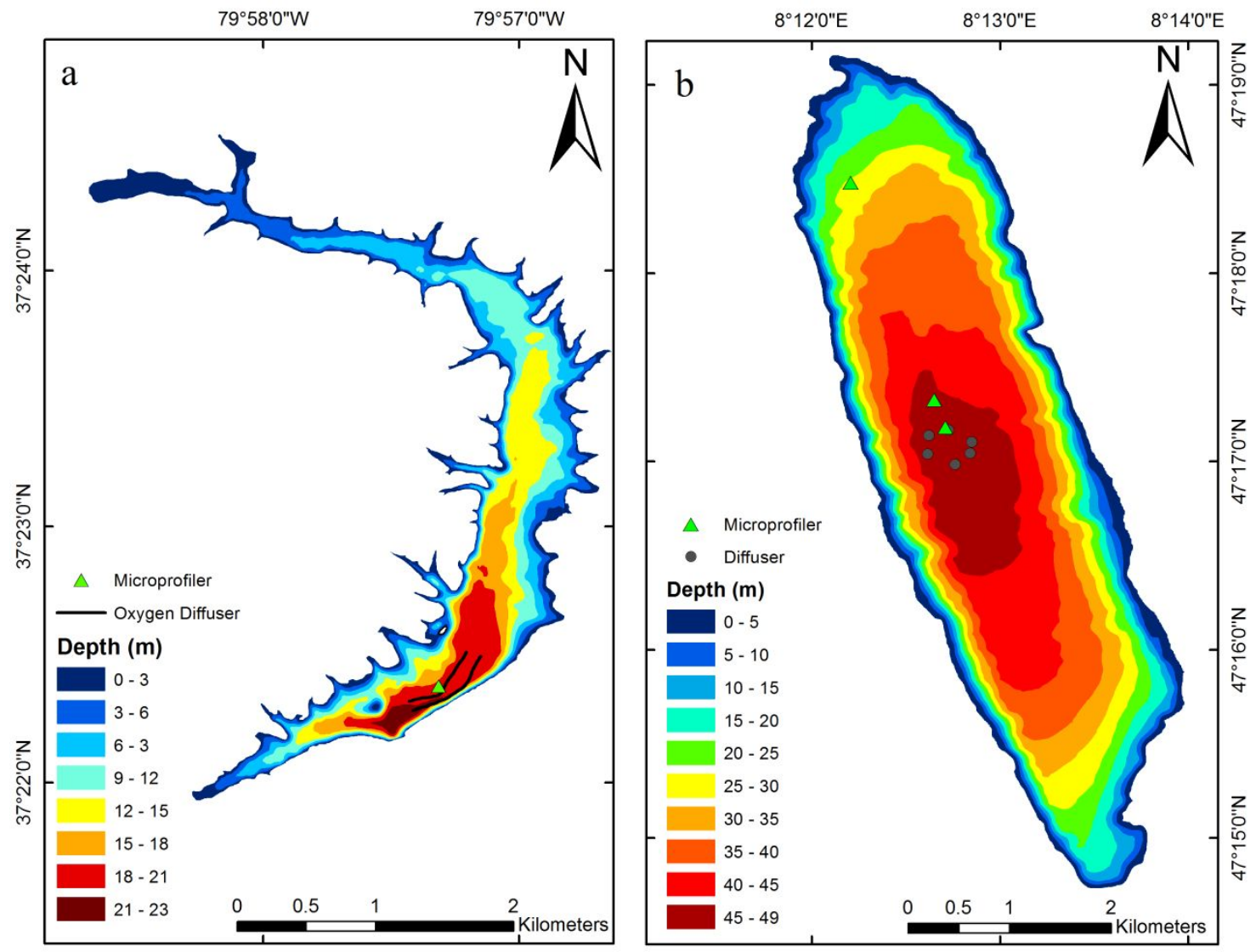

Figure 1. Bathymetric maps of (a) Carvins Cove Reservoir (CCR) and (b) Lake Hallwil (LH).

168 Locations of the linear diffusers in CCR and the circular diffusers in LH are shown, as well as the

169 deployed positions of the microprofiler. Note that one of the six diffuser symbols in LH is partially

170 obscured by a symbol for the microprofiler.

172 DO microprofiles were measured in situ in two oxygenated lakes: Lake Hallwil and Carvins Cove

173 Reservoir (Figure 1). Lake Hallwil (LH) is located north of Lucerne, Switzerland, on the Swiss 
174 Plateau, and is primarily used for recreational purposes. It has a maximum depth of $48 \mathrm{~m}$, a surface 175 area of $9.95 \mathrm{~km}^{2}$, a volume of $285 \times 10^{6} \mathrm{~m}^{3}$ and is at an elevation of $449 \mathrm{~m}$ above mean sea level. 176 Carvins Cove Reservoir (CCR) is a water-supply reservoir for the City of Roanoke in southwest 177 Virginia, USA. CCR has a maximum depth of $22 \mathrm{~m}$, a surface area of $2.5 \mathrm{~km}^{2}$, a volume of $24 \times 10^{6}$ $178 \mathrm{~m}^{3}$ and is at an elevation of $357 \mathrm{~m}$ above mean sea level.

\subsection{Microprofile Data Collection}

181 A microprofiler (MP4; Unisense A/S) was deployed for periods of $\sim 2$ to $\sim 5$ days at three locations 182 along the main axis of LH (as shown in Figure 1) from 24 May to 1 June 2012. The microprofiler 183 was deployed at one location in CCR (also shown in Figure 1) from 26 May to 2 June 2013. While 184 deployed, the microprofiler was equipped with a Clark-type oxygen microsensor (Unisense OX185 100), as well as a thermocouple temperature sensor (Unisense TP-200). Measurements were made 186 in triplicate at a sampling rate of $1 \mathrm{~Hz}$ at the following vertical resolution: $10 \mathrm{~mm}$ resolution from $187100 \mathrm{~mm}$ to $10 \mathrm{~mm}$ above the SWI, $1 \mathrm{~mm}$ resolution from $10 \mathrm{~mm}$ to $5 \mathrm{~mm}$ above the SWI, and 0.1 $188 \mathrm{~mm}$ resolution from $5 \mathrm{~mm}$ above the SWI to $5 \mathrm{~mm}$ below the SWI. The single oxygen microsensor was used to obtain three measurements in rapid succession at each vertical depth position; these

190 triplicate measurements were averaged prior to modelling the data. This protocol resulted in 191 complete microprofiles of both DO and temperature measured across the SWI every 55 minutes.

193 The SWI location was visually determined by examining each microprofile to identify the linear 194 region in the DBL and the slope change associated with the porosity difference between the water 195 and sediment. The standard deviation of the triplicate DO measurements taken at each point in 196 the microprofiles was used to further identify and confirm the correct location of the SWI, as 197 standard deviation of DO measurements should decrease as the microsensor approaches the SWI ${ }^{21}$.

\subsection{Sediment Kinetics}

200 Sediment DO kinetics is typically described using zero-order, first-order, or Monod (sometimes 201 referred to as Michaelis-Menten) kinetic models. Zero-order and first-order kinetic models are 202 shown below in equations 1 and $2^{30}$. These model formulations account for molecular diffusion 203 of DO through the sediment porewater and consumption of DO within the sediment. Rather than 204 trying to account for the numerous chemical and biological processes that consume DO in the 
sediment, DO consumption is assumed to be adequately represented using an all-encompassing rate constant, or in the case of the Monod model, two constants. Monod kinetics have the effect of becoming zero-order or first-order depending on DO concentrations.

209 The study by House ${ }^{30}$ showed that zero-order and Monod models described DO kinetics within 210 the sediment equally well, although zero-order often agreed slightly better. Olinde ${ }^{36}$ also showed 211 that despite the additional fitting parameter offered by Monod kinetics, it does not result in a 212 substantially improved fit to DO microprofiles measured during sediment core incubations. Given 213 these observations and to minimize model complexity, the Monod kinetic model was not employed 214 in this study. By applying a finite shift to the zero-order and first-order kinetic models (equations 2151 and 2), the transient numerical solutions can be obtained (equations 3 and 4) as:

where $C$ represents DO concentration, $t$ is time, $D_{s}$ is the effective diffusion coefficient of DO in 224 the sediment (where $D_{s}=\varphi D_{m}, \varphi$ is sediment porosity and $D_{m}$ is the molecular diffusion coefficient 225 of DO in water), $z$ is depth below the SWI, $k_{0}$ is a zero-order rate constant, and $k_{l}$ is a first-order 226 rate constant. The superscript $i$ denotes the profile number in the time series of profiles, and the subscript $n$ represents depth in the porewater DO profile, where the positive upward direction is 228 towards the sediment.

229 The models were coded using Matlab ${ }^{37}$. They were initialized with the first profile in the series of 230 microprofiles measured at each deployment, using only the portion of the profile at and below the 231 SWI. Measured DO concentrations below $3 \mu \mathrm{mol} \mathrm{L}^{-1}$ were forced to zero, as concentrations below 232 this level become difficult to discern from zero due to microsensor capabilities; furthermore, the 233 depth at which DO is $<3 \mu \mathrm{mol} \mathrm{L}^{-1}\left(0.096 \mathrm{mg} \mathrm{L}^{-1}\right)$ has been defined as the depth of maximum DO 
234 penetration in previous studies (e.g., Bryant et al. ${ }^{35}$ ). The model assumes constant temperature 235 over the series of profiles, as well as constant $\varphi$ with sediment depth. This results in a constant 236 value of $D_{s}$ for a given series of profiles. Measurements of $\varphi$ were obtained from sediment cores 237 collected from both CCR and LH during field studies conducted prior to the current study, 238 following Dalsgaard et $a l^{38}$. The $\varphi$ values in the sediment of CCR and LH were 0.96 and 0.94 , 239 respectively ${ }^{21}$. The upper boundary condition is the measured DO concentration at the SWI. This 240 study adopts four methods of characterising boundary conditions, denoted by ZOZF, ZONF, FOZF 241 and FONF. ZOZF (zero-order, zero-flux) and ZONF (zero-order, negative-flux) adopt zero-order 242 kinetics, while FOZF (first-order, zero-flux) and FONF (first-order, negative-flux) adopt first243 order kinetics. The lower boundary condition for ZOZF and FOZF is the traditional zero-flux 244 boundary condition (equation 5) with the lower boundary located where the measurement 245 terminates $(5 \mathrm{~mm})$ :

$$
D_{s} \frac{\partial C}{\partial z}=0 \mathrm{gm}^{-2} d^{-1}
$$

The lower boundary condition for ZONF and FONF is a negative-flux boundary condition (equation 6) with the lower boundary located where DO concentration goes to zero in each profile, representing a fixed value of reduced species flux based on the general value Müller et $a l^{23}$. obtained for 11 eutrophic lakes:

$$
D_{s} \frac{\partial C}{\partial z}=-0.25 g^{-2} d^{-1}
$$

The benthic flux of reduced substance is set to a fixed value rather than being treated as a second 257 fitting parameter to ensure the stability of the kinetic model and to keep the model simple. Because equations 1 and 2 are expressed in terms of DO, we assume that the upward flux of reduced compounds is rapidly oxidized by DO and that the result could be reasonably expressed as an 260 upward "negative" flux of DO at the depth where the DO concentration becomes zero for each 261 profile. To ensure a stable solution that does not oscillate, the time-step $(\Delta t)$ is made sufficiently 262 small so that the diffusion number ( $\lambda$, equation 7$)$ is less than $0.25^{39}$. 
$264 \lambda=\frac{D_{m} \Delta t}{\Delta z^{2}}$

Since the $\Delta t$ necessary to achieve $\lambda<0.25$ is much smaller than the time interval between two consecutive microprofiles measured in the field $(\Delta t=1 \mathrm{~s}$ in the model versus $\Delta t \approx 55 \mathrm{~min}$ in field measurements), the DO concentration at the SWI used as the upper boundary condition for the modeled profiles is determined by linearly interpolating between measured DO concentrations at the SWI as needed. After the DO concentrations for every $\Delta t(1 \mathrm{~s})$ have been simulated, the DO

272 profile $\mathrm{i}$, where $t^{i}$ is the time when profile i starts being measured, and $t^{i+1}$ is the time when profile

273 i+1 starts being measured.

The models were evaluated using rate constants from $1-2000\left(\mathrm{mg} \mathrm{L}^{-1} \mathrm{~d}^{-1}\right.$ for zero-order kinetic models and $\mathrm{d}^{-1}$ for first-order kinetic models), whose range was selected based on reported values

277 from previous studies $21,22,28,30$. The best-fit value for the kinetic rate constant is the value that 278 minimizes the root mean square error (RMSE).

$$
R M S E=\sqrt{\frac{\sum\left(C_{o b s}-C_{S i m}\right)^{2}}{p}}
$$

280 for each microprofile, where $C_{O b s}$ is observed DO concentration, $C_{S i m}$ is simulated DO 281 concentration, and $p$ is the total number of microprofile data points among all depths and profiles.

282 After calculating the best-fit $k_{0}$ or $k_{l}$ for all profiles in one series, the average values of the best-fit $283 k_{0}$ and $k_{1}$ were applied as the best-fit kinetic rate constants for the full series of microprofiles; these 284 globally averaged values were deemed most suitable due to the high variability in the profile285 specific rate constants (as shown in Figure S5 - S8). The averaged best-fit $k_{0}$ and $k_{1}$ are used for additional analysis described in Section 2.4.

\subsection{Model versus field data comparisons}

290 Using the fitted $k_{0}$ and $k_{l}$ from each series of profiles, the sediment-side DO flux is calculated from 291 the simulated profiles and compared to sediment-side DO flux calculated from the measured microprofiles. DO flux $\left(J_{O_{2}}\right)$ was estimated as: 


$$
\mathrm{J}_{O 2}=\mathrm{D}_{s} \frac{\Delta C}{\Delta z}
$$

where $\Delta C / \Delta z$ is the DO concentration gradient immediately below the SWI. To compare how well the modeled data agree with the field data, the relative error and the normalized RMSE are calculated using equations 10 and 11 , where $\mathrm{N}$ is the number of profiles, $J_{O_{2}, \text { Sim }}$ is the simulated DO flux, $J_{\mathrm{O}_{2}, \mathrm{Obs}}$ is the observed DO flux, and the subscript $i$ denotes profile number in the time series of profiles:

$$
\text { Relative error }=\frac{J_{o_{2}, S i m_{i}}-J_{o_{2}, O b s_{i}}}{J_{o_{2}, o b s_{i}}}
$$

\subsection{RESULTS and DISCUSSION}

\section{$307 \quad 3.1 \quad$ Fitted Rate Constants}

308 The fitted values of $k_{0}$ for ZOZF and ZONF, the fitted values of $k_{1}$ for FOZF and FONF, as well 309 as the corresponding average RMSE values for each series of microprofiles, are displayed in Table 310 1. Values for $k_{0}$ and $k_{1}$ range from $120-510 \mathrm{mg} \mathrm{L}^{-1} \mathrm{~d}^{-1}$ and $90-400 \mathrm{~d}^{-1}$ in $\mathrm{LH}$, while values for $311 \mathrm{CCR}$ are lower at $50-60 \mathrm{mg} \mathrm{L}^{-1} \mathrm{~d}^{-1}$ and $60-80 \mathrm{~d}^{-1}$. Differences in the fitted rate constants 312 between the two study sites are likely attributable to differences in the sediment composition 313 between the two lakes, including the amount of labile organic matter and mineral composition. 314 Labile carbon, reduced metals, and other chemical species exert a demand for DO within the 315 sediment, and larger quantities of any of these oxygen-consuming species would result in an 316 increase in the observed rate constant. The large range in fitted rate constants within LH is likewise 317 attributable to spatial variability in sediment composition within LH itself. The first and third 318 deployments were both in the central, deepest portion of LH ( 42 m depth and $\sim 300 \mathrm{~m}$ apart) and 319 yielded similar best-fit rate constants. The second deployment, though still in the hypolimnion, 320 was at a shallower location ( $25 \mathrm{~m}$ depth) and has higher fitted rate constants. Sediment in the 321 shallower portions of LH is likely to have more labile carbon in the sediment than deeper portions 322 of the lake ${ }^{21}$, since settling organic matter has less time to be oxidized in the water column before 
323 reaching the sediment. A similar observation was made in a study of Lake Gevena ${ }^{40}$, a Swiss lake

324 where sediment DO uptake declines with increasing lake depth due to decreased rates of organic 325 matter settling and sediment surface mineralization with greater depth. Additionally, the LH 326 bubble-plume oxygenation system is located in the deepest portion of the lake, within $300 \mathrm{~m}$ of 327 the first and third microprofiler deployments ( $\mathrm{LH}-1$ and $\mathrm{LH}-3$, respectively). This oxygenation 328 system, which has been in operation for roughly 30 years, would further enhance oxidation of 329 settling organic matter in the water column by increasing the availability of DO in the water 330 column, as well as by satisfying oxygen demand exerted by the nearby sediment. It has been 331 shown that the organic content of sediment in LH has been significantly decreased by 332 oxygenation ${ }^{23,41}$. Thus, it seems reasonable that the fitted rate constant should be higher in the 333 shallower regions of the lake, which are also farther from the oxygenation system. Temperature 334 may play a minor role in the increased best-fit rate constants at the shallower site, as temperatures 335 measured near the SWI were only about $1^{\circ} \mathrm{C}$ warmer at this shallower location.

337 In both the CCR data set and the LH data sets, ZONF and FONF (with a negative-flux lower 338 boundary condition) appear to fit much better than ZOZF or FOZF (zero-flux lower boundary 339 condition) as evidenced by the distinctly lower RMSE values (Table 1). Most of the observed 340 profiles also agree much better visually with the simulated profiles of ZONF and FONF than with 341 those of ZOZF or FOZF in all data sets, as shown in Figures 2 and 3. It should be noted that in 342 CCR, some profiles simulated by FOZF fit the field profiles very well while other profiles 343 simulated by FOZF have rather high RMSEs, which is the reason why FOZF outperforms FONF 344 in Table 2, but not Table 1. Considering this visual comparison and the relatively lower RMSE 345 values, the negative-flux lower boundary condition appears to be more appropriate for describing 346 DO consumption close to the lower boundary of the sediment for both LH and CCR. This provides 347 strong evidence supporting the description of hypolimnetic DO consumption in the model of 348 Müller et al. ${ }^{23}$ Moreover, these new results indicate that it is possible to simulate DO consumption 349 in the upper sediment by modeling the lumped zero-order or first-order reaction and the reduced 350 species fluxes. 

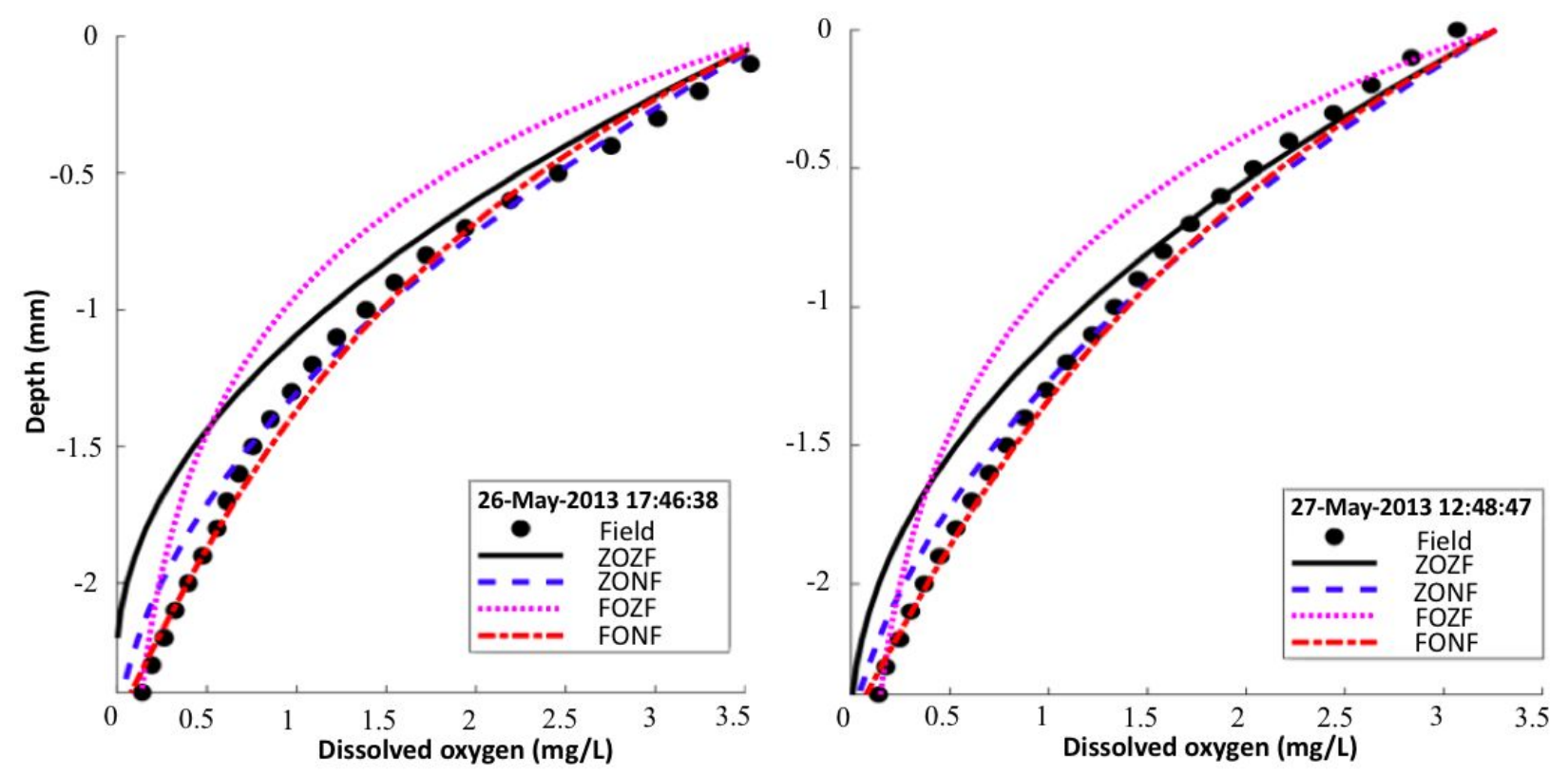

Figure 2. Characteristic DO microprofiles comparing field data to simulations by the four methods (ZOZF, ZONF, FOZF, and FONF) in Carvins Cove Reservoir (CCR).

354
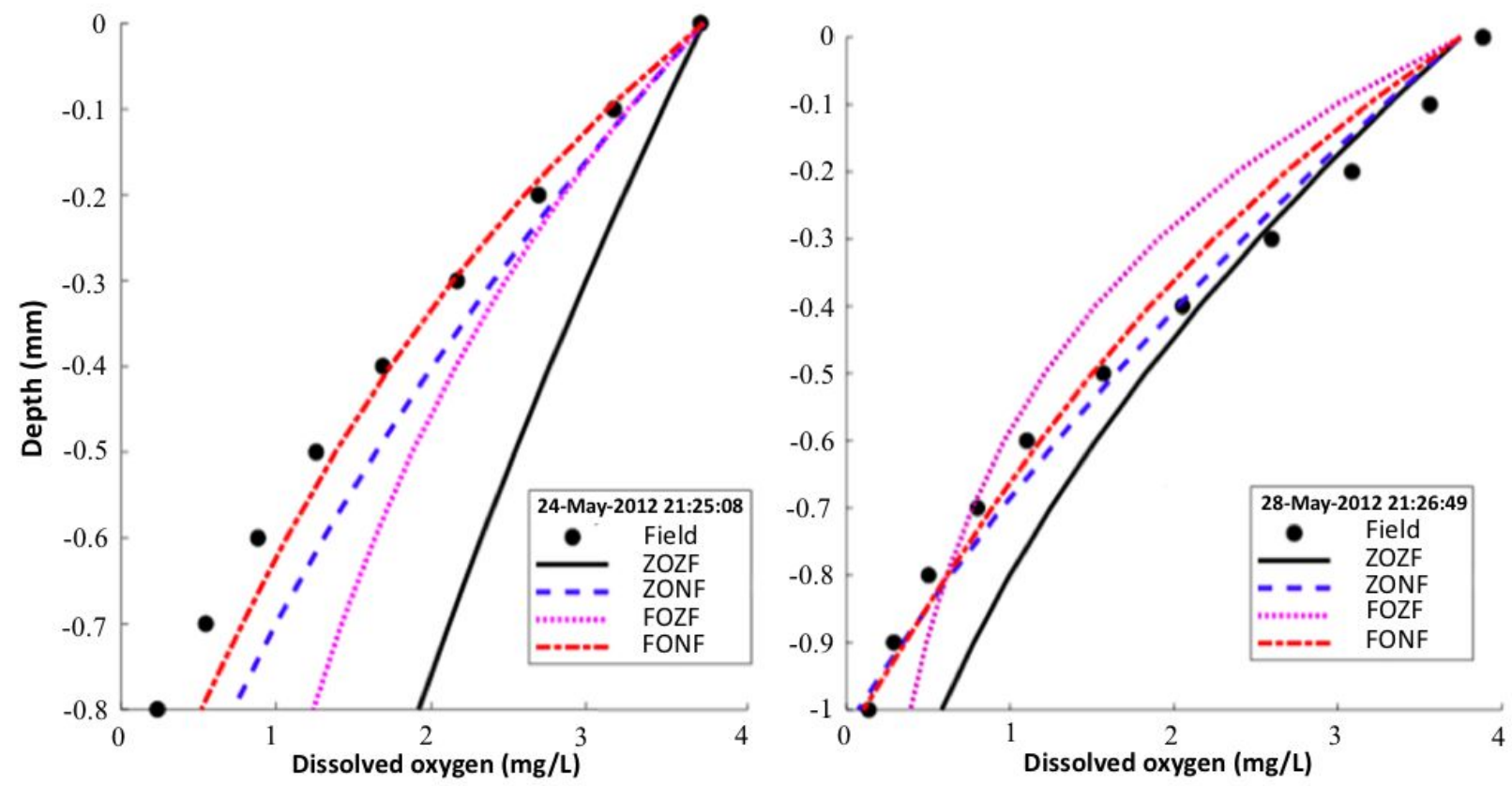

356 Figure 3. Characteristic DO microprofiles comparing field data to simulations by the four methods (ZOZF, ZONF, FOZF, and FONF) in Lake Hallwil (LH). 
Table 1. Summary of fitted kinetic rate constants and average root mean square error (RMSE) with lower RMSEs between ZOZF/ZONF and lower RMSEs between FOZF/FONF shown in bold.

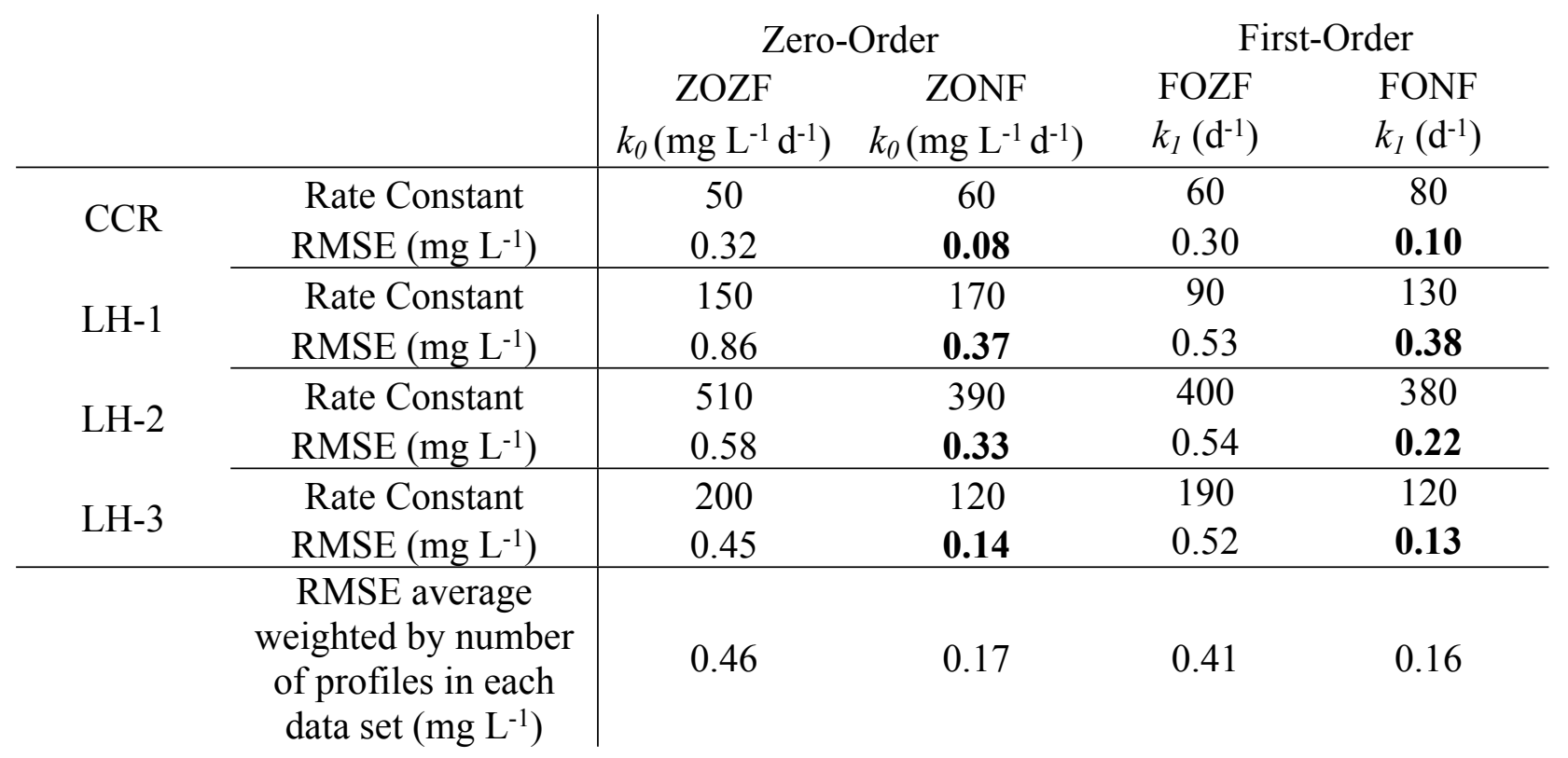

Table 2. Percentage of each fitting method with the best performance among all 320 profiles (combined total for $\mathrm{LH}$ and $\mathrm{CCR}$ ) based on RMSE value for each individual profile (higher percentages between ZOZF/ZONF and higher percentages between FOZF/FONF are shown in bold)

\begin{tabular}{c|c|c|c|c|}
\cline { 2 - 5 } & $\begin{array}{c}\text { ZOZF } \\
(\mathbf{\%})\end{array}$ & $\begin{array}{c}\text { ZONF } \\
(\mathbf{\%})\end{array}$ & $\begin{array}{c}\text { FOZF } \\
(\mathbf{\%})\end{array}$ & $\begin{array}{c}\text { FONF } \\
(\mathbf{\%})\end{array}$ \\
\hline CCR & 13 & $\mathbf{5 7}$ & $\mathbf{1 6}$ & 14 \\
LH-1 & 9 & $\mathbf{2 3}$ & $\mathbf{2 3}$ & $\mathbf{4 5}$ \\
LH-2 & 13 & $\mathbf{3 3}$ & 11 & $\mathbf{4 3}$ \\
LH-3 & 8 & $\mathbf{4 7}$ & 6 & $\mathbf{3 9}$
\end{tabular}

Differences between the simulated and observed profiles are likely a result of adopting the globally averaged rate constants and also the interpolation necessary to force the model. Since the field

371 data only has direct DO measurements at the SWI roughly every 55 minutes, any fluctuation in

372 DO concentrations at the SWI occurring on a shorter time scale are not represented in the model 373 forcing. As mentioned in the methods section, the model time-step is one second ( $\Delta t=1 \mathrm{~s})$ to 374 ensure stable solutions, which is much shorter than the $\sim 55$-min period between two consecutive 375 in situ microprofiles. If higher frequency data were available to force the model, the agreement 
376 between the simulated and observed profiles would likely improve. In some microprofiles (e.g., 377 the profiles in Figure 2), the simulated DO concentration at depth $=0 \mathrm{~mm}$ is not equal to that of 378 the observations. This is also related to interpolation of field profiles and reflects the time379 consuming microprofile measurement process.

381 Despite some differences in the methods used for determining the best-fit rate constants, the fitted 382 values generally compare quite well with the preliminary CCR study by Olinde ${ }^{35}$. Olinde found 383 the best-fit $k_{0}$ to be $36 \pm 10 \mathrm{mg} \mathrm{L}^{-1} \mathrm{~d}^{-1}$ at $4^{\circ} \mathrm{C}$ and $130 \pm 66 \mathrm{mg} \mathrm{L}^{-1} \mathrm{~d}^{-1}$ at $20^{\circ} \mathrm{C}$, while the best-fit $k_{1}$ 384 was $34 \pm 12 \mathrm{~d}^{-1}$ at $4{ }^{\circ} \mathrm{C}$ and $170 \pm 68 \mathrm{~d}^{-1}$ at $20^{\circ} \mathrm{C}$. The fitted CCR values obtained in the current 385 study (Table 2), measured at an in situ temperature of $\sim 7.5^{\circ} \mathrm{C}$ agree nicely with the range from this 386 preliminary study by Olinde ${ }^{35}$.

Given the differences in methods and variable sediment composition and microbial community structure $^{43}$, the magnitude of the optimized zero-order rate constant for CCR agrees with $k_{0}$ 390 reported by Rasmussen and Jørgensen ${ }^{21}\left(83 \mathrm{mg} \mathrm{L}^{-1} \mathrm{~d}^{-1}\right)$ and House ${ }^{28}\left(9.7-44 \mathrm{mg} \mathrm{L}^{-1} \mathrm{~d}^{-1}\right)$. The 391 fitted $k_{0}$ for LH are higher than those reported by Rasmussen and Jørgensen ${ }^{21}$ or House $^{28}$ but are 392 still within a reasonable range; in comparison, Hall et al. ${ }^{29}$ reported a $k_{0}$ that is an order of 393 magnitude higher than those from LH (1750 mg L-1 $\mathrm{d}^{-1}$ versus $\left.120-510 \mathrm{mg} \mathrm{L}^{-1} \mathrm{~d}^{-1}\right)$. It should 394 also be taken into consideration that the negative-flux lower boundary condition in ZONF and 395 FONF assumes an instantaneous reaction, while in natural water bodies the oxidation of reduced 396 species is clearly not instantaneous but only gradually reduces the concentrations of reduced 397 species over the distance of diffusion. Since the concentration gradient of reduced species in the 398 upper sediments is extremely complex to characterize, the proposed simplification is reasonable 399 but may lead to larger $k_{0}$ and $k_{l}$ estimates.

\subsection{DO Flux Comparisons}



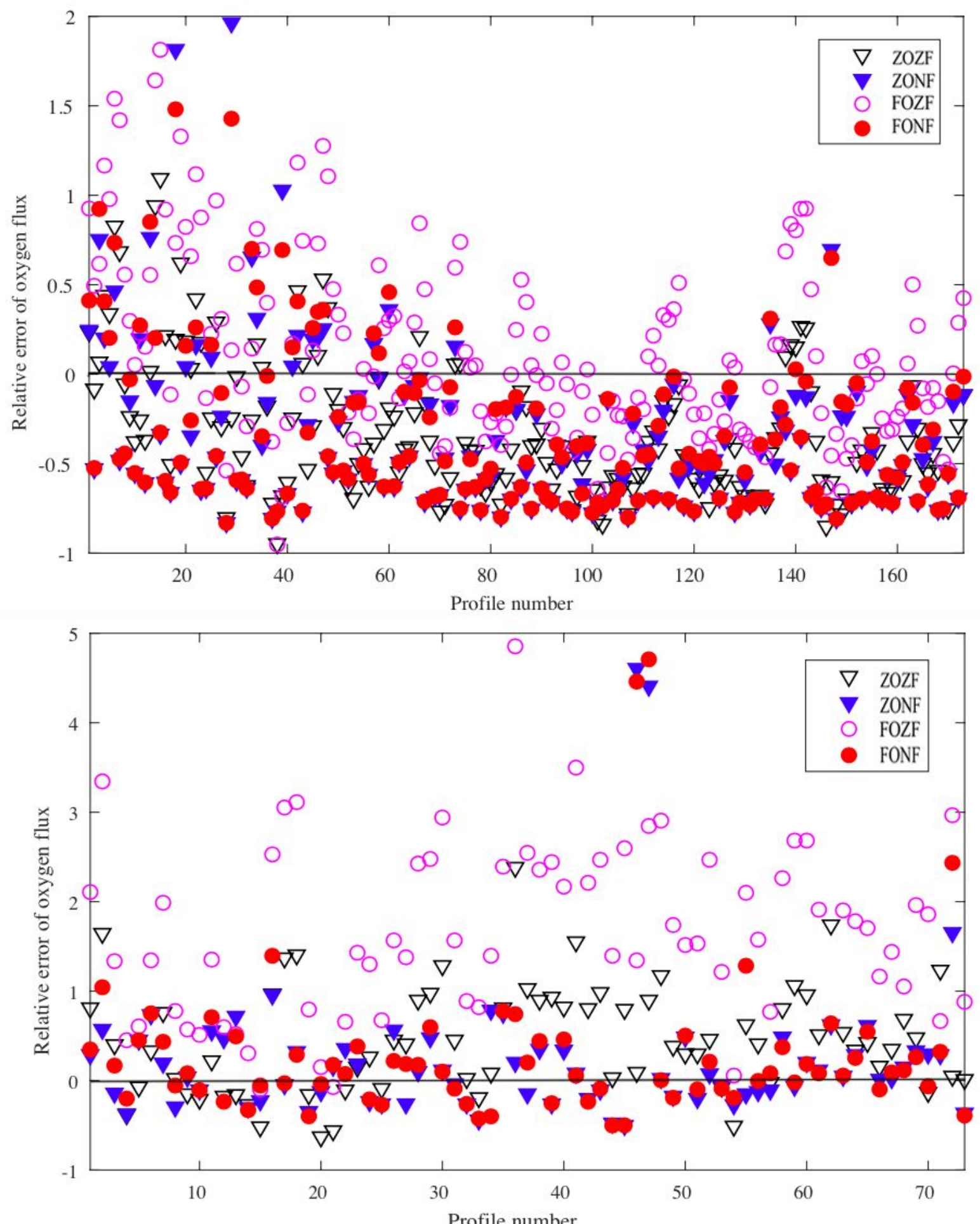

Profile number

404 Figure 4. Relative error of the sediment-side DO fluxes at the sediment-water interface

405 (SWI) in the CCR microprofiler deployment between 2013 May 26 and 2013 June 02 
406

407

408

409

410

411

412

413

414

415

416

417

418

419

420

421

422

423

424

425

426

427

428

429

430

431

432

433

434

435

436

(upper plot) and the third microprofiler deployment in LH (LH - 3) between 2012 May 28 and 2012 June 01 (lower plot).

Sediment-side DO fluxes at the SWI calculated from the simulated DO profiles were used to estimate $J_{O 2}$, which were then compared to field measurements. The first-order kinetics with zeroflux boundary condition (FOZF) typically overestimates the DO flux. The sediment-side DO flux calculated by negative-flux lower boundary conditions (ZONF, FONF) agrees better than zeroflux lower boundary conditions (ZOZF, FOZF) with the field data based on both visual inspection and the normalized RMSE values weighted by the number of profiles in each data set as shown in Figure 4 (and also Table S2 and Table S3). Due to the overestimation of FOZF, the normalized RMSE of DO flux calculated by FOZF is around twice the normalized RMSE of negative-flux lower boundary condition (ZONF, FONF) for both CCR and LH. This increased RMSE, along with Section 3.1 results, lend more support to using the negative-flux lower boundary condition over the zero-flux lower boundary condition when modeling DO flux on the sediment side of SWI in LH and CCR. In addition, ZONF seems to be the best method for LH, yielding the lowest RMSE. Another aspect to be considered is that Monod kinetics, a rate expression that is often used to describe microbial growth and single substrate degradation, have the effect of being zeroorder when the concentration is much greater than the Monod half-saturation constant while being first-order when the concentration is much less than the half-saturation constant ${ }^{39}$. Because virtually all microbial DO consumption processes have a half-saturation constant near or below the detection limit of the microsensor ${ }^{44}\left(3 \mu \mathrm{mol} \mathrm{L}^{-1}\right)$, it is not surprising that zero-order kinetics yield more reliable results.

DO fluxes at the SWI of some profiles have a normalized RMSE of more than $200 \%$, which indicates that fluxes calculated from these simulated microprofiles, primarily by FOZF, do not adequately reproduce the actual measured fluxes. Müller et al. ${ }^{23}$ assumed the reaction of DO with organic matter to be a first-order kinetic reaction, which indicates that the negative-flux lower boundary condition has only been studied and verified with first-order sediment kinetics. This may explain why FONF performs much better than FOZF while ZONF does not outperform ZOZF in DO flux comparison such as those discussed in Section 3.1. Additional sources of discrepancy may be due to the measured DO fluxes being subject to measurement errors at the SWI, especially 
437 related to the determination of the exact SWI depth level, and the simulated DO fluxes being 438 influenced by errors arising from the model and numerical solutions.

440 Both the simulated and observed sets of DO fluxes have significant implications for lake and 441 reservoir management. The sediment-side DO fluxes are closely related to the water-side DO 442 fluxes, which can also be derived from the kinetic rate constants based on empirical equations ${ }^{19,25}$. 443 The comparison of the simulated and observed DO fluxes indicates that the simulated ones are 444 more reliable than the observed ones because the simulated ones make use of all measured 445 microprofiles. For these reasons, comprehensive microprofiling time-series paired with the model 446 described in this study can provide a useful new tool in monitoring sediment DO fluxes for 447 managing water quality and ecosystem health in lakes and reservoirs.

\subsection{Limitation of the approach}

450 A model of DO transport and consumption in freshwater sediments has been fit to DO 451 microprofiles measured in situ, using zero-order and first-order kinetic models with zero-flux and 452 negative-flux lower boundary conditions to determine the appropriate kinetic order and the 453 appropriate bottom boundary condition. While the results support the inclusion of a flux of 454 reduced species propagating upwards from deeper within the sediments, and thereby contributing 455 to part of the overall sediment DO consumption and corresponding DO flux estimates, additional 456 work is necessary. An even longer time-series of microprofiles may be obtained to make the 457 analysis more robust. The overestimation of DO flux in some microprofiles is also likely to be a 458 result of the interpolation necessary to force the model. With a higher-frequency data set to force 459 the model, the agreement between observed and simulated microprofiles is expected to improve, 460 which should, in turn, improve the fluxes calculated from the simulated profiles. If possible, 461 placing focus on profiling immediately across the SWI and into the upper sediment (thereby 462 excluding a majority of the overlying water column) would allow for considerably decreased 463 profiling times, thereby minimising the difference between actual measurements and model time 464 steps. In addition, the negative-flux value is fixed in this study; however, Müller et al. ${ }^{23}$ suggested 465 that values may vary among different lakes and even across different seasons and/or locations 466 within the same lake. Further research is needed to better characterize the value of sediment DO 467 fluxes in these complex aquatic systems using simplified, yet accurate models. 


\section{ASSOCIATED CONTENT}

\section{Supporting Information}

471 The Supporting Information contains additional details on previous studies investigating DO 472 kinetics in sediment porewater (Table S1), comparison between normalized RMSE of DO flux 473 using the four methods (ZOZF, ZONF, FOZF, and FONF; Table S2), percentage of profiles whose 474 sediment-side DO flux have relative error less than \pm 0.5 and \pm 1.0 (Table S3), DO concentration at 475 the SWI for each site (Figure S1 - S4), kinetic rate constants of each profile for each site (Figures 476 S5 - S8), in situ and simulated DO profiles by ZOZF, ZONF, FOZF and FONF in CCR and LH 477 (Figure S9-S169).

479 AUTHOR INFORMATION

480 Corresponding Author

481 *E-mail: jcl@vt.edu; Phone: (540) 2310836.

\section{ORCID}

484 Lee D. Bryant: 0000-0002-1783-6942

485 Chengwang Lei: 0000-0002-8175-4781

486 John C. Little: 0000-0003-2965-9557

487 Alfred Wüest: 0000-0001-7984-0368

488 Notes

489 The authors declare no competing financial interest.

\section{ACKNOWLEDGEMENTS}

492 This research was primarily supported by U.S. National Science Foundation grant CBET 1033514. 493 We would like to thank the Western Virginia Water Authority for access to Carvins Cove 494 Reservoir and Kanton Aargau for access to Lake Hallwil during the field campaigns. We also are 495 grateful to Michael Schurter of Eawag, Arno Stöckli of Kanton Aargau, Scott Socolofsky and 496 Maryam Rezvani of Texas A\&M University, and Christina Urbanczyk for their valuable assistance 497 during the field campaigns. 


\section{REFERENCES}

$500 \quad$ 1. Conley, D. J.; Björke, S.; Bonsdorf, E.; Carstensen, J.; Destouni, G.; Gustafsson, B. G.;

501 Hietanan, S.; Kortekaas, M.; Kuosa, H.; Meier, H. E. M.; Müller-Karulis, B.; Nordberg, K.;

502 Norkko, A.; Nürnberg, G.; Pitkänen, H.; Rabalais, N. N.; Rosenberg, R.; Savchuk, O. P.; Slomp,

503 C.P.; Voss, M.; Wulff, F.; Zillén, L. Hypoxia-related processes in the Baltic Sea. Environ. Sci.

504 Technol. 2009, 43 (10), 3412-3420.

5052 2. Li, Q.; Bi, S.; Ji, G. Determination of strongly reducing substances in sediment. Environ.

506 Sci. Technol. 2003, 37 (24), 5727-5731.

$507 \quad 3 . \quad$ Beutel, M. W.; Leonard, T. M.; Dent, S. R.; Moore, B. C.; Effects of aerobic and

508 anaerobic conditions on $\mathrm{P}, \mathrm{N}, \mathrm{Fe}, \mathrm{Mn}$, and $\mathrm{Hg}$ accumulation in waters overlaying profundal

509 sediments of an oligo-mesotrophic lake. Water Res. 2008, 42 (8-9), 1953-1962.

510 4. Huttunen, J. T.; Väisänen, T. S.; Hellsten, S. K.; Martikainen, P. J. Methane fluxes at the

511 sediment-water interface in some boreal lakes and reservoirs. Boreal Environ. Res. 2006, 11 (1)

512 27-34.

513 5. Davison, W. Iron and manganese in lakes. Earth Sci. Rev. 1993, 34, 119-163.

$514 \quad$ 6. Wang, H.; Dai, M.; Liu, J.; Kao, S.; Zhang, C.; Cai, W.; Wang, G.; Qian, W.; Zhao, M.;

515 Sun, Z. Eutrophication-driven hypoxia in the East China Sea off the Changjiang Estuary.

516 Environ. Sci. Technol. 2016, 50, (5), 2255-2263.

$517 \quad 7 . \quad$ Funkey, C. P.; Conley, D. J.; Reuss, N. S.; Humborg, C.; Jilbert, T.; Slomp, C. P. Hypoxia 518 sustains cyanobacteria blooms in the Baltic Sea. Environ. Sci. Technol. 2014, 48 (5), 2598-2602.

519 8. Gao, Y.; Cornwell, J. C.; Stoecker, D. K.; Owens, M. S. Influence of cyanobacteria blooms 520 on sediment biogeochemistry and nutrient fluxes. Limnol. Oceanogr. 2014, 59 (3), 959-971.

5219 9. Wu, R. S. S.; Zhou, B. S.; Randall, D. J.; Woo, N. Y. S.; Lam, P. K. S. Aquatic hypoxia is 522 an endocrine disruptor and impairs fish reproduction. Environ. Sci. Technol. 2003, 37 (6), $1137-$

$530 \quad$ 12. Trottet, A.; Wilson, B.; Xin, G. S. W.; George, C.; Casten, L.; Schmoker, C.; Rawi, N. S. 1141.

10. Gantzer, P. A.; Bryant, L. D.; Little, J. C. Controlling soluble iron and manganese in a water-supply reservoir using hypolimnetic oxygenation. Water Res. 2009, 43 (5), 1285-1294.

11. Cheng, Y. S.; McDonald, J. D.; Kracko, D.; Irvin, C. N.; Zhou, Y.; Pierce, R. H.; Henry, M. S.; Bourdelaisa, A.; Naar, J.; Baden D. G. Concentration and particle size of airborne toxic algae (Brevetoxin) derived from ocean read tide events. Environ. Sci. Technol. 2005, 39 (10), 3443-3449. B. N.; Siew, M. C.; Larsen, O.; Eikaas, H. S.; Tun, K.; Drillet, G. Resting stage of plankton diversity from Singapore coastal water: Implications for harmful algae blooms and coastal management. Environ. Manage. 2018, 61 (2),275-290.

13. Schindler, D. W.; Carpenter, S. R.; Chapra, S. C.; Hecky, R. E.; Orihel, D.M. Reducing phosphorus to curb lake eutrophication is a success, Environ. Sci. Technol. 2016, 50 (17), 89238929.

14. Singleton, V. L.; Little, J. Designing hypolimnetic aeration and oxygenation systems - A review. Environ. Sci. Technol. 2006, 40 (24), 7512-7520.

15. Kleeberg, A.; Herzog, C.; Hupfer, M. Redox sensitivity of iron in phosphorus binding does not impede lake restoration. Water Res. 2013, 47 (3), 1491-1502.

541 16. Testa, J. M.; Kemp, W. M. Hypoxia-induced shifts in nitrogen and phosphorus cycling in 542 Chesapeake Bay. Limnol. Oceanogr. 2012, 57 (3), 835-850. 
17. Gächter, R.; Wehrli, B. Ten years of artificial mixing and oxygenation: no effect on the internal phosphorus loading of two eutrophic lakes. Environ. Sci. Technol. 1998, 32, 3659-3665. 18. Matzinger, A.; Müller, B.; Niederhauser, P.; Schmid, M.; Wüest, A.; Hypolimnetic oxygen consumption by sediment-based reduced substances in former eutrophic lakes. Limnol. Oceanogr. 2010, 55, 2073-2084.

548 19. Higashino, M.; Gantzer, C.; Stefan, H. Unsteady diffusional mass transfer at the 549 sediment/water interface: Theory and significance for SOD measurement. Water Res. 2004, 38 $550 \quad(1), 1-12$.

551 20. Beutel M. W. Hypolimnetic anoxia and sediment oxygen demand in California drinking Water Reservoirs, Lake. Resevir. Manage., 2003, 19 (3), 208-221, DOI: 10.1080/07438140309354086

21. Bryant, L. D.; McGinnis, D. F.; Lorrai C.; Brand A.; Little J. C.; Wüest A. Evaluating oxygen fluxes using microprofiles from both sides of the sediment-water interface, Limnol. Oceanogr. Methods, 2010a, 8, 610-627. doi:10.4319/lom.2010.8.0610. coastal sediment: role of molecular diffusion. Mar. Ecol. Pro. Ser. 1992, 81 (3), 289-303. 23. Müller, B.; Bryant, L. D.; Matzinger, A.; Wüest, A. Hypolimnetic oxygen depletion in eutrophic lakes. Environ. Sci. Technol. 2012, 46 (18), 9964-9971.

24. O'Connor, B.; Hondzo, M.; and Harvey, J. Incorporating both physical and kinetic limitations in quantifying dissolved oxygen flux to aquatic sediments. J. Environ. Eng. 2009, 135 (12), 1304-1314.

25. Bierlein, K. A.; Rezvani, M..; Socolofsky, S. A.; Bryant, L. D.; Wüest, A.;Little, J. C. Increased sediment oxygen flux in lakes and reservoirs: The impact of hypolimnetic oxygenation. Water Resour. Res. 2017, 53 (6), 4876-4890, doi:10.1002/2016WR019850

26. Larsen, S. J.; Kilminster, K. L.; Mantovanelli, A.; Goss, Z. J.; Evans, G. C.; Bryant, L. D.; McGinnis D. F. Artificially oxygenating the Swan River estuary increases dissolved oxygen concentrations in the water and at the sediment interface. Ecol. Eng. 2019, 128, 112-121

27. Brewer, W; Abernathy, A. R.; Paynter, M. J. B. Oxygen consumption by freshwater sediments. Water Res. 1977, 11 (5), 471-473.

28. Beutel, M.; Hannoun, I.; Pasek, J.; Kavanagh, K. B. Evaluation of hypolimnetic oxygen demand in a large eutrophic raw water reservoir, San Vicente Reservoir, Calif. J. Environ. Eng. 2007, 133 (2), 130-138.

29. Hall, P. O. J.; Anderson, L. G.; van der Loeff, M. M. R.; Sundby, B.; Westerlund, S. F. G. Oxygen uptake kinetics in the benthic boundary layer. Limnol. Oceanogr. 1989, 34 (4), 734-746.

30. House, W. Factors influencing the extent and development of the oxic zone in sediments. Biogeochem. 2003, 63 (3), 317-334.

31. Bryant, L. D.; Lorrai, C.; McGinnis, D. F.; Brand, A.; Wüest, A.; Little, J. C. Variable sediment oxygen uptake in response to dynamic forcing. Limnol. Oceanogr. 2010b, 55 (2), $950-$ 964.

32. Berg, P.; Risgaard-Petersen, N.; Rysgaard, S. Interpretation of measured concentration profiles in sediment pore water. Limnol. Oceanogr. 1998, 43(7), 1500-1510.

33. Brady, D. C.; Testa, J. M; Di Toro, D. M.; Boynton, W. R.; Kemp, W. M. Sediment flux modelling: Calibration and application for coastal systems, Est. Coa. She. Sci. 2013. 117, 107124. 
34. Kristensen, E.; Hansen, T.; Matthieu, D.; Banta, G.T.; Cintia, C. Q. Contrasting effects of the polychaetes Marenzelleris viridis and Nereis diversicolor on benthic metabolism and solute transport in coastal sediment, Mar. Ecol. Prog. Ser. 2011, 425, 125-139.

590 35. Bryant, L. D.; Hsu-Kim, H.; Gantzer, P. A.; Little, J.C. Solving the problem at the source: 591 Controlling Mn release at the sediment-water interface via hypolimnetic oxygenation. Water Res. 2011, 45(19), 6381-6392. https://doi.org/10.1016/j.watres.2011.09.030

593 36. Olinde, L. J. Sediment oxygen demand kinetics, Master's Thesis, Virginia Polytechnic Institute and State University. 2007

595 37. MATLAB and Statistics Toolbox Release 2014, The MathWorks, Inc., Natick, 596 Massachusetts, United States.

597 38. Bryant, L. D.; P. A. Gantzer.; Little, J. C. Increased sediment oxygen uptake caused by 598 oxygenation-induced hypolimnetic mixing. Water Res. 2011, 45 (12), 3692-3703.

599 39. Dalsgaard, T.; Nielsen L. P.; Brotas, V.; Viaroli, P.; Underwood, G.; Nedwell, D. B.; 600 Sundbäck, K.; Rysgaard, S.; Miles, A.; Bartoli, M.; Dong, L.; Thornton, D. C. O.; Ottosen, L. D. 601 M.; Castaldelli, G.; Risgaard-Petersen, N. 2000. Protocol Handbook for NICE - Nitrogen Cycling 602 in Estuaries: A Project under the EU Research Programme: Marine Science and Technology 603 (MAST). National Environmental Research Institute, Silkeborg, Denmark. 604 http://www2.dmu.dk/LakeandEstuarineEcology/nice/NICE handbook.pdf

605 40. Chapra, S. C. Surface water-quality modeling. McGraw-Hill, Boston, Mass, USA. 1997, 606 pg 214.

607 41. Schwefel, R.; Steinsberger, T.; Bouffard, D., Bryant, L.; Müller, B.; Wüest, A. Using 608 small-scale measurements to estimate hypolimnetic oxygen depletion in a deep lake. Limnol. 609 Oceanogr. 2018, 63 (S1), S54-S67. https://doi.org/10.1002//no.10723

610 42. Liu, C.; Zachara, J. M. Uncertainties of monod kinetics parameters nonlinearly estimated 611 from batch experiments. Environ. Sci. Technol. 2001, 35 (1), 133-141.

612 43. Bryant, L. D.; Little, J. C.; Bürgmann, H. Response of sediment microbe community 613 structure in a freshwater reservoir to manipulations in oxygen availability. FEMS Microbiol. Ecol. 614 2012, 80, 248-263. http://doi.org/10.1111/j.1574-6941.2011.01290.x

$61544 . \quad$ Stadler, L. B.; Love, N. G. Oxygen half-saturation constants for pharmaceuticals in 616 activated sludge and microbial community activity under varied oxygen levels. Environ. Sci.

617 Technol. 2019, 53 (4), 1918-1927. 